\title{
The Geneva-Copenhagen survey of the solar neighbourhood
}

\section{Improved distances, ages, and kinematics ${ }^{\star}$}

\author{
J. Holmberg ${ }^{1}$, B. Nordström ${ }^{1}$, and J. Andersen ${ }^{1,2}$ \\ 1 The Niels Bohr Institute, Astronomy Group, Juliane Maries Vej 30, 2100 Copenhagen, Denmark \\ e-mail: [birgitta; johan] @astro.ku.dk \\ 2 Nordic Optical Telescope Scientific Association, Apartado 474, 38700 Santa Cruz de La Palma, Spain
}

Received 20 October 2008 / Accepted 19 December 2008

\section{ABSTRACT}

\begin{abstract}
Context. Ages, chemical compositions, velocity vectors, and Galactic orbits for stars in the solar neighbourhood are fundamental test data for models of Galactic evolution. The Geneva-Copenhagen Survey of the Solar Neighbourhood (Nordström et al. 2004; GCS), a magnitude-complete, kinematically unbiased sample of 16682 nearby $\mathrm{F}$ and $\mathrm{G}$ dwarfs, is the largest available sample with complete data for stars with ages spanning that of the disk.

Aims. We aim to improve the accuracy of the GCS data by implementing the recent revision of the Hipparcos parallaxes.

Methods. The new parallaxes yield improved astrometric distances for 12506 stars in the GCS. We also use the parallaxes to verify the distance calibration for $u v b y \beta$ photometry by Holmberg et al. (2007, A\&A, 475, 519; GCS II). We add new selection criteria to exclude evolved cool stars giving unreliable results and derive distances for 3580 stars with large parallax errors or not observed by Hipparcos. We also check the GCS II scales of $T_{\text {eff }}$ and $[\mathrm{Fe} / \mathrm{H}]$ and find no need for change.

Results. Introducing the new distances, we recompute $M_{V}$ for 16086 stars, and $U, V, W$, and Galactic orbital parameters for the 13520 stars that also have radial-velocity measurements. We also recompute stellar ages from the Padova stellar evolution models used in GCS I-II, using the new values of $M_{V}$, and compare them with ages from the Yale-Yonsei and Victoria-Regina models. Finally, we compare the observed age-velocity relation in $W$ with three simulated disk heating scenarios to show the potential of the data. Conclusions. With these revisions, the basic data for the GCS stars should now be as reliable as is possible with existing techniques. Further improvement must await consolidation of the $T_{\text {eff }}$ scale from angular diameters and fluxes, and the Gaia trigonometric parallaxes. We discuss the conditions for improving computed stellar ages from new input data, and for distinguishing different disk heating scenarios from data sets of the size and precision of the GCS.
\end{abstract}

Key words. solar neighborhood - Galaxy: disk - Galaxy: stellar content - Galaxy: kinematics and dynamics - Galaxy: evolution stars: fundamental parameters

\section{Introduction}

A wide range of observations can be used to test models of the evolution of disk galaxies such as the Milky Way. The most detailed and complete data consist of the ages, chemical compositions, space motions, and Galactic orbits of stars in the solar neighbourhood, but a large sample of stars is needed, covering the full possible range of ages in the Galactic disk. Moreover, the sample selection criteria must be well-defined, and the calibrations by which the astrophysical parameters are derived from observations must be well understood.

The Geneva-Copenhagen survey of the Solar neighbourhood (GCS) was designed to fulfil these criteria. Nordström et al. (2004; GCS I) presented ages, metallicities, and complete kinematic information for over 14000 nearby $\mathrm{F}$ and $\mathrm{G}$ dwarfs, based on $u v b y \beta$ photometry, Hipparcos parallaxes, and $\sim 63000$ new, accurate radial-velocity observations. Ages were computed by the Bayesian technique of Jørgensen \& Lindegren (2005).

This data base has been used in a large number of studies of the evolution of the Galactic disk. Some of these also discuss the derived parameters, notably $T_{\text {eff }},[\mathrm{Fe} / \mathrm{H}]$, and ages. In

^ Full Table 1 is only available in electronic form at the CDS via anonymous ftp to cdsarc.u-strasbg.fr (130.79.128.5) or via http://cdsweb.u-strasbg.fr/cgi-bin/qcat?J/A+A/501/941
Holmberg et al. (2007; GCS II), we therefore derived new calibrations of $u v b y \beta$ photometry into $T_{\text {eff }}$, based on the robust calibration of $V-K$ into $T_{\text {eff }}$ and available $V-K$ photometry, and for $[\mathrm{Fe} / \mathrm{H}]$ based on recent high-resolution spectroscopy. Significant revision was found necessary for the $T_{\text {eff }}$ calibration; for $[\mathrm{Fe} / \mathrm{H}]$ the changes were only minor. Ages were also recomputed with the new data; they were $\sim 10 \%$ lower than the GCS I ages for the oldest stars, and in excellent agreement with the independent results of Takeda et al. (2007). All the revised parameters are given in the GCS II catalogue ${ }^{1}$. In the end, however, the revised data showed no material change in the fundamental relations between age, metallicity, and kinematics.

From the detailed discussion in GCS II it is clear that the dominant source of uncertainty in determining isochrone ages for FG dwarfs is the $T_{\text {eff }}$ scale and - linked to it - that of $[\mathrm{Fe} / \mathrm{H}]$. In short, a dichotomy exists between $T_{\text {eff }}$ as derived from the excitation equilibrium of iron, and from photometry via the infrared flux method (IRFM) and ultimately anchored in bolometric fluxes and stellar angular diameters. There are also some differences between various implementations of the IRFM, as discussed below. The weak point in the first method is the use

\footnotetext{
1 We regret that a considerable delay occurred in submitting the GCS II data to the CDS; they are now available at http://cdsarc. u-strasbg.fr/viz-bin/Cat?V/117A
} 
of static, 1D LTE models to approximate real stellar surfaces, in the second the few calibration stars with precise data currently available, especially at lower metallicity. We see little prospect for real progress on the $T_{\text {eff }}$ calibration until these basic weaknesses are alleviated.

However, the re-reduction of the Hipparcos data by van Leeuwen (2007) has substantially improved the parallax values. The stellar distances directly affect the computed space motions and Galactic orbits and are usually the major source of uncertainty; via $M_{V}$ they also enter in the age calculations. We decided, therefore, to implement the new parallaxes in the GCS, use them to improve the photometric distances that we use when the parallax errors are large, and recompute the ages, space motions, and Galactic orbits of the GCS stars accordingly.

This paper describes our procedures and results (the on-line version of the catalogue at the CDS will contain the complete data as presented in GCS I, but give the $T_{\text {eff }}$ and $[\mathrm{Fe} / \mathrm{H}]$ values of GCS II and the new parameters derived in this paper). In preparation, we have checked the GCS II $T_{\text {eff }}$ and $[\mathrm{Fe} / \mathrm{H}]$ calibrations with precise angular diameters (Sect. 3.1) and highresolution spectroscopy published since that paper (Sect. 3.2). We then compare ages derived from the new data with three different sets of stellar evolution models (Sect. 4) and comment on the procedures for reliable age determinations (Sect. 6.1). Finally, in Sect. 6.3 we compare the observed age-velocity relation with simulations of three disk heating scenarios.

\section{Improved distances}

The re-reduction of the raw Hipparcos data by van Leeuwen (2007) reduced the parallax errors by a factor $\sim 1.5$ on average, a substantial improvement on the original results. We have therefore substituted the new parallaxes in the GCS data base. This is the primary source of distance information for the vast majority (now 12506) of our stars.

In GCS I we used the distance calibrations by Crawford (1975) for $F$ stars and by Olsen (1984) for $G$ stars to compute photometric distances for stars without good parallaxes. Comparison with the most accurate Hipparcos parallaxes showed these photometric distances to be accurate to $13 \%$, so they were preferred when the parallax error was larger. 3- $\sigma$ discrepancies between the two distance estimates were used to flag suspected binaries, giants, and stars with spectral peculiarities relative to normal dwarfs.

In GCS II we reexamined the calibrations and found that, while they gave good distances within the stated accuracy as shown in GCS I, systematic effects as a function of colour were present. We therefore took advantage of the accurate Hipparcos parallaxes to derive a new distance calibration, using only stars with $\sigma_{M_{V}}<0.1 \mathrm{mag}$ and no indication of binarity in GCS I. As shown in GCS II (Fig. 10), the new calibration was markedly more reliable when compared to the most accurate Hipparcos parallaxes.

The Crawford (1975) and Olsen (1984) calibrations were designed for dwarf stars only. The same holds for the GCS II calibration due to a lack of evolved stars in the calibration sample: Because the GCS was designed as a survey of dwarfs, most substantially evolved stars were excluded a priori, based on the gravity-sensitive $\delta \mathrm{c}_{1}$ index. All three calibrations were therefore used with confidence in GCS I and GCS II for all stars without good parallaxes.

With the improved Hipparcos parallaxes, however, a larger sample of evolved cool stars with parallax errors below $13 \%$ is available. It reveals a clear systematic error in the photometric
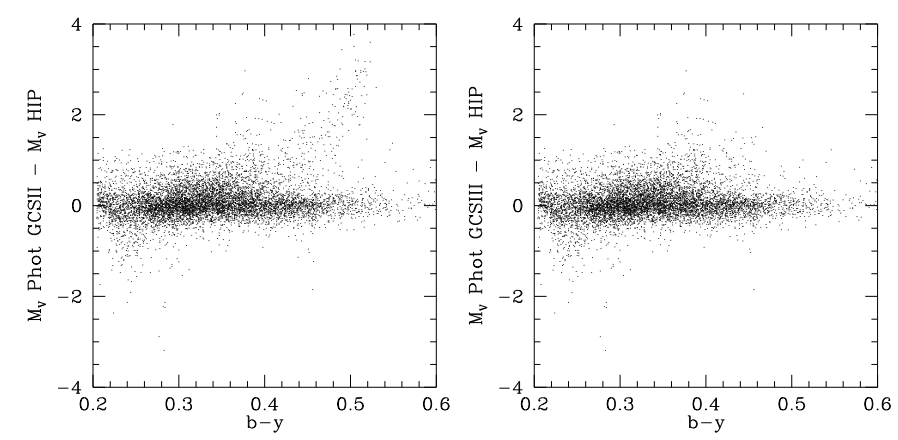

Fig. 1. Differences between $M_{V}$ as determined from the $u v b y$ photometry with the GCS II calibration, and from the new Hipparcos parallaxes. Left: full GCS sample. Right: restricted colour range (see text).

distances for such stars. Close inspection of the data indicates that the $\delta \mathrm{m}_{1}$ index can be used as an effective criterion to eliminate the unreliable evolved stars from the dwarfs in the GCS. We find that, to a very large extent, stars with $b-y>0.41$ and $\delta m_{1}>0.06$ (150 stars) are subgiants that were not excluded by the GCS photometric selection criteria. Outside this restricted range the general calibration derived in GCS II remains valid and yields excellent, unbiased distance estimates; in the GCS III catalogue it is used for 3580 stars.

Figure 1 compares $M_{V}$ as derived from the GCS II calibration, with and without the colour restriction derived above; the improvement in the red range is obvious. We further note that one general result of using the new Hipparcos parallaxes is to increase the number of suspected binaries; the total number of known or suspected binaries in the GCS is now 6228 , or an overall binary fraction of $37 \%$.

\section{Re-checking the other GCS II calibrations}

In this section, we briefly compare the temperature and metallicity scales derived in GCS II with observational data published since that paper was completed.

\section{1. $T_{\mathrm{eff}}$}

The pros and cons of photometric vs. spectroscopic temperature determinations; our reasons for preferring the former; and our new $u v b y \beta$ calibration were discussed in detail in GCS II. That discussion will not be repeated, but we here briefly note that a few new angular diameters have been published. From them, a "true" effective temperature can be derived through the relation:

$f_{\mathrm{bol}}=\frac{\phi^{2}}{4} \sigma T_{\mathrm{eff}}^{4}$,

where $f_{\text {bol }}$ is the bolometric flux, $\phi$ the angular diameter of the star and $\sigma$ the Stefan-Boltzmann constant.

Ramírez \& Meléndez (2005) combined published stellar diameters, mostly from the VLTI, with bolometric flux measurements to derive $T_{\text {eff }}$ for 10 dwarfs and 2 sub-giants. We can now supplement the four high-accuracy measurements of their sample with four new diameters from the CHARA (Baines et al. 2008; Boyajian et al. 2008) and SUSI interferometers (North et al. 2007, 2009). This gives us a sample of nine stars with diameters accurate to $2 \%$ or better, and with good temperatures in GCS II as well. The mean difference is $\left\langle T_{\mathrm{eff}}^{\text {ours }}-T_{\mathrm{eff}}^{\text {dia }}\right\rangle=23 \pm 19 \mathrm{~K}$, with a standard deviation (s.d.) of $57 \mathrm{~K}$ (see Fig. 2). 


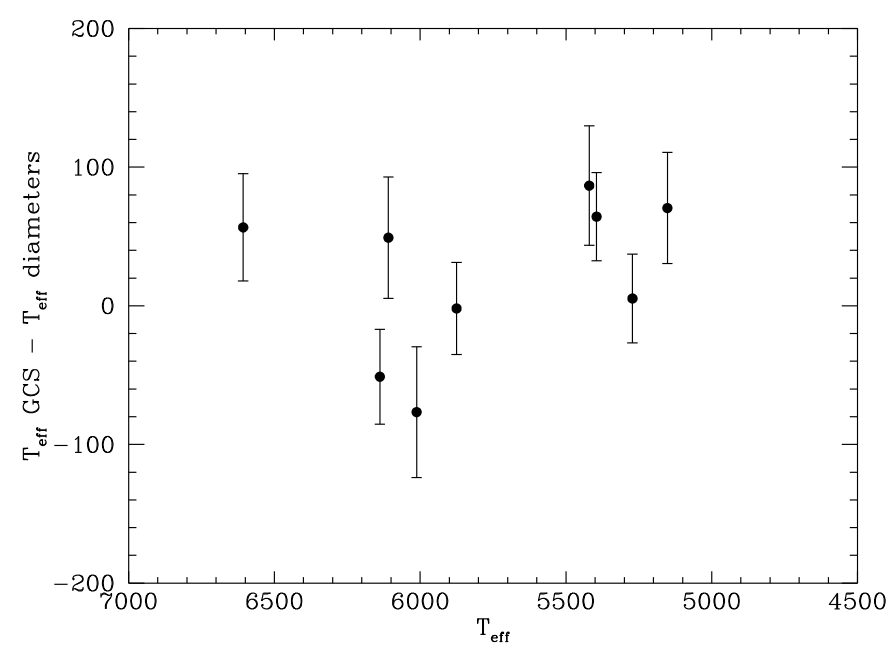

Fig. 2. $T_{\text {eff }}$ as determined from recent angular diameter measurements vs. $T_{\text {eff }}$ from GCS II, based on $u v b y \beta$ photometry. The error bars correspond only to the errors in the diameters (better than 2\%) and fluxes (assumed to be 3\%).

Recent large spectroscopic studies of GCS stars include Sousa et al. (2008), who determined $T_{\text {eff }}$ from the excitation balance in iron. For the 330 stars in common, the mean difference is $\left\langle T_{\mathrm{eff}}^{\text {Sousa }}-T_{\mathrm{eff}}^{\text {ours }}\right\rangle=54 \mathrm{~K}$, with a s.d. of $71 \mathrm{~K}$.

Jenkins et al. (2008) also give spectroscopic $[\mathrm{Fe} / \mathrm{H}]$ values for 161 GCS stars, but use a photometric temperature calibration from Blackwell \& Lynas-Gray (1994). The mean difference is $\left\langle T_{\text {eff }}^{\text {Jenkins }}-T_{\text {eff }}^{\text {ours }}\right\rangle=43 \mathrm{~K}$, with a s.d. of $93 \mathrm{~K}$, with systematically high $T_{\text {eff }}$ near $5500 \mathrm{~K}$ and lower for hotter and cooler stars.

We also compare our $T_{\text {eff }}$ values to the recent implementation of the IRFM by Casagrande et al. (2006), who find $T_{\text {eff }} \mathrm{S}$ closer to the spectroscopic scale. We confirm this result for the sample of 57 stars in common with the GCS: The mean difference is $\left\langle T_{\text {eff }}^{\text {Casagrande }}-T_{\text {eff }}^{\text {ours }}\right\rangle=55 \mathrm{~K}$, with a s.d. of $91 \mathrm{~K}$. Their $T_{\text {eff }}$ is systematically low near $5300 \mathrm{~K}$, and high for both hotter and cooler stars, i.e. the reverse of the trend of the Jenkins data. This was found also by Casagrande et al. (2006) when comparing with the di Benedetto (1998) scale, which we used in GCS II to calibrate our own $u v b y \beta$ scale; the mean difference was $\left\langle T_{\text {eff }}^{\text {Casagrande }}-T_{\text {eff }}^{\text {diBenedetto }}\right\rangle=50 \pm 50 \mathrm{~K}$.

In summary, the data appearing after GCS II are consistent with the results derived there and give no reason for changing the GCS II temperature calibration.

\section{2. $[\mathrm{Fe} / \mathrm{H}]$}

$[\mathrm{Fe} / \mathrm{H}]$ serves both as a tracer of the chemical evolution of disk stars and as input parameter to the computation of stellar ages from theoretical isochrones. Comparing the $[\mathrm{Fe} / \mathrm{H}]$ values from GCS II with those of Sousa et al. (2008), we find $\left\langle[\mathrm{Fe} / \mathrm{H}]^{\text {Sousa }}-\right.$ $\left.[\mathrm{Fe} / \mathrm{H}]^{\text {ours }}\right\rangle=0.05 \mathrm{dex}$, with a s.d. of 0.10 dex. Here there is a very clear systematic trend with high spectroscopic $[\mathrm{Fe} / \mathrm{H}]$ for hotter stars.

For Jenkins et al. (2008), the difference is $\left\langle[\mathrm{Fe} / \mathrm{H}]^{\text {Jenkins }}-[\mathrm{Fe} / \mathrm{H}]^{\text {ours }}\right\rangle=0.06 \mathrm{dex}$, with a s.d. of $0.13 \mathrm{dex}$; a systematic trend is seen here as well, mirroring that in $T_{\text {eff }}$ from the same source.

The above offsets precisely reflect the differences in the adopted $T_{\text {eff }}$ values, as discussed in detail in GCS II. Until these ambiguities are resolved in a definitive manner, we find no reason to modify the $[\mathrm{Fe} / \mathrm{H}]$ calibration and the individual values given in GCS II.

\section{Stellar ages}

With the new distance data $\left(M_{V}, \sigma_{M_{V}}\right)$ and the GCS II values for $T_{\text {eff }}$ and $[\mathrm{Fe} / \mathrm{H}]$, we have computed new ages from the Padova models used in GCS II (Girardi et al. 2000; Salasnich et al. 2000). The same temperature corrections were applied to the isochrones as in GCS II to achieve consistency between the observed and computed lower main sequences, as the $T_{\text {eff }}$ and $[\mathrm{Fe} / \mathrm{H}]$ scales remain the same.

The ages and their uncertainties were also computed with the same techniques as in GCS I and GCS II (Jørgensen \& Lindegren 2005) - now the standard method in the field, but with one modification: For a few nearby stars, the new Hipparcos parallaxes are so precise that the corresponding $M_{V}$ fixes the age of stars in certain parts of the HR diagram with unrealistically high precision. We have therefore imposed a floor of $0.05 \mathrm{mag}$ for the error in $M_{V}$ before computing the ages.

The results are compared with those from GCS II in Fig. 3 (left). As seen, the overall consistency is very good, but occasionally the changes in $M_{V}$ lead to substantial changes in the ages. We consider the new ages to be superior to those in GCS II and give them in Table 1 along with the derived upper and lower $1-\sigma$ confidence limits.

In order to assess the model dependence of the resulting ages, we have compared our results from the Padova models to ages derived from both the Yale-Yonsei (Demarque et al. 2004) and Victoria-Regina sets of model computations (VandenBerg et al. 2006). In each case, temperature corrections appropriate for each isochrone set were applied to ensure that the unevolved models and observed main sequences agreed at all metallicities, as described in detail in GCS I and II. The corrections applied to the $Y-Y$ and $V-R$ sets were found to be very similar to those applied to the Padova models in GCS II. For $Y-Y$ somewhat larger, and for $V-R$ somewhat smaller, temperature corrections were needed, but of the same general form, with larger corrections for more metal-poor models.

Figure 3 compares the results. As will be seen, the new (GCS III) ages are in excellent agreement with those from the $V-R$ models, while the $Y-Y$ models have a small positive offset for young stars and give $\sim 10 \%$ lower ages for stars older than the Sun. Note that observational errors result in age estimates larger than the WMAP figure of 13.7 Gyr for some old stars; truncating the models artificially at that age would have biased the determination of these ages and their errors, as well as the mean age of the oldest stars.

\section{Results}

The revised distances have been used to compute new values of the $U, V$, and $W$ components of the space motion, using the same conventions as in GCS I. Some of these velocities differ by several $\mathrm{km} \mathrm{s}^{-1}$ from those given in GCS I, so we have also recomputed the Galactic orbital parameters $R_{\mathrm{p}}, R_{\mathrm{a}}, e$, and $z_{\max }$, using a Galactic potential similar to that used in GCS I (Flynn et al. 1996). All the revised quantities are given in Table 1.

\section{Discussion}

In the following, we discuss first the criteria and methods for determining isochrone ages, then review some of the basic relations between age, metallicity, and kinematics in the light of 

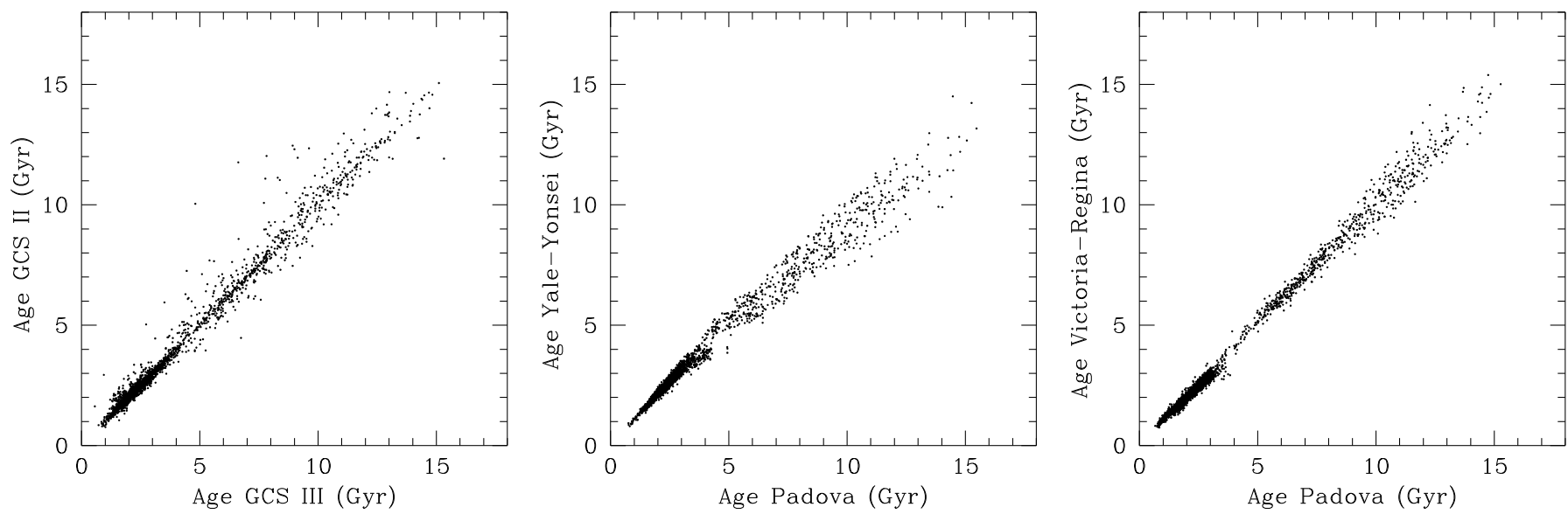

Fig. 3. (Left:) GCS III vs. GCS II ages. Middle: GCS III ages (Padova isochrones) vs. ages from Yale-Yonsei models. Right: same for Victoria-Regina models. Single stars with $\sigma_{\text {Age }}<25 \%$ in each set (all panels).

Table 1. Sample listing of the recomputed parameters for the first five stars in the GCS catalogue.

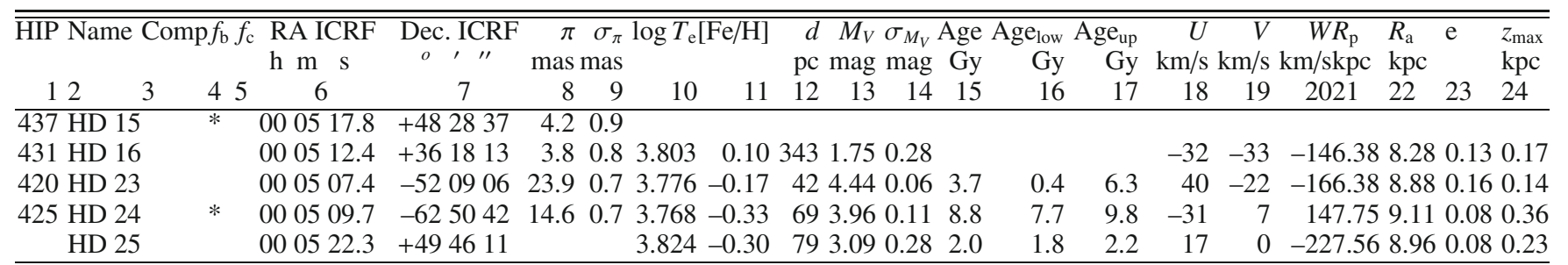

$f_{\mathrm{b}}$ marks stars suspected to be binaries. $f_{\mathrm{c}}=1$ identifies Hyades and Coma stars with photometry as given in GCS I; $f_{\mathrm{c}}=2$ identifies Hyades/Coma stars for which the standard photometry by Crawford \& Perry (1966) and Crawford \& Barnes (1969) is used in GCS II and here. $\pi$ and $\sigma_{\pi}$ are the new Hipparcos parallaxes and their errors (van Leeuwen 2007), and $\mathrm{Age}_{\mathrm{low}}$ and $\mathrm{Age}_{\mathrm{up}}$ are the lower and upper 1- $\sigma$ confidence limits on the computed age. (The full table is only available in electronic form at the CDS).

the new data. As indicated by the comparisons made above, any changes relative to the results of GCS I and II are expected to be minor, so the discussion will be relatively brief.

In order to obtain the clearest possible picture of the evolution of the local Galactic disk, we restrict the sample discussed below to stars that have ages better than $25 \%$ and trigonometric parallaxes better than $13 \%$. We also require the stars to have no indication of binarity, either by having at least two concordant modern radial-velocity measurements, no binary flag from the Hipparcos parallax reduction, or concordant astrometric and photometric parallaxes. This leaves us with a sample of 2626 stars.

\subsection{Computing reliable isochrone ages}

The discussion above may suggest that computing stellar ages from isochrones is now straightforward, and recomputing ages with a favourite choice of $T_{\text {eff }}$ and/or $[\mathrm{Fe} / \mathrm{H}]$ has become common practice. The different results that emerge are the subject of much discussion, but before progress is claimed, it will be useful to recall the following points:

- at the basic conceptual level, isochrone ages are determined by placing a star in the HR diagram from its observed $T_{\text {eff }}$ and $M_{v}$ and interpolating in a set of isochrones for the observed metallicity;

- the derived age depends most sensitively on $T_{\text {eff }}$, so any change in the scale of effective temperature will affect the derived ages in a systematic and probably metallicitydependent way;
- changing $[\mathrm{Fe} / \mathrm{H}]$ changes the $Z$ value of the models, hence the ages. Non-solar abundance ratios require particular attention; e.g., neglect of the change in $[\alpha / \mathrm{Fe}]$ in stars of different $[\mathrm{Fe} / \mathrm{H}]$ leads directly to an age-metallicity relation of the expected slope (see GCS II);

- whatever scale of $T_{\text {eff }}$ and/or $[\mathrm{Fe} / \mathrm{H}]$ is adopted, it must be verified that the observed and computed unevolved main sequences $\left(M_{V}>5.5\right)$ are consistent before meaningful ages can be derived;

- finally, an age computation technique that takes the observational biases into account and returns reliable estimates of the uncertainties is essential, such as that by Jørgensen \& Lindegren (2005). Ages are usually plotted as points, but real uncertainties are usually large, as discussed in detail in GCS I.

Real stars, of course, have single, consistent values of $T_{\text {eff }}$, $[\mathrm{Fe} / \mathrm{H}]$, and age. Thus, one cannot combine "observed" $T_{\text {eff }}$ values, spectroscopic $[\mathrm{Fe} / \mathrm{H}]$ determinations assuming another $T_{\text {eff }}$ scale, and models using a different mix of elements and definition of $T_{\text {eff }}$ and expect to get reliable ages. Unless it is verified that observations and models are consistent for unevolved stars, new age calculations do not necessarily also mean progress.

\subsection{Age-metallicity diagram}

The relationship between age and metallicity for stars in the solar neighbourhood - the age-metallicity relation (AMR) - is probably the most popular diagnostic diagram for comparing galactic 

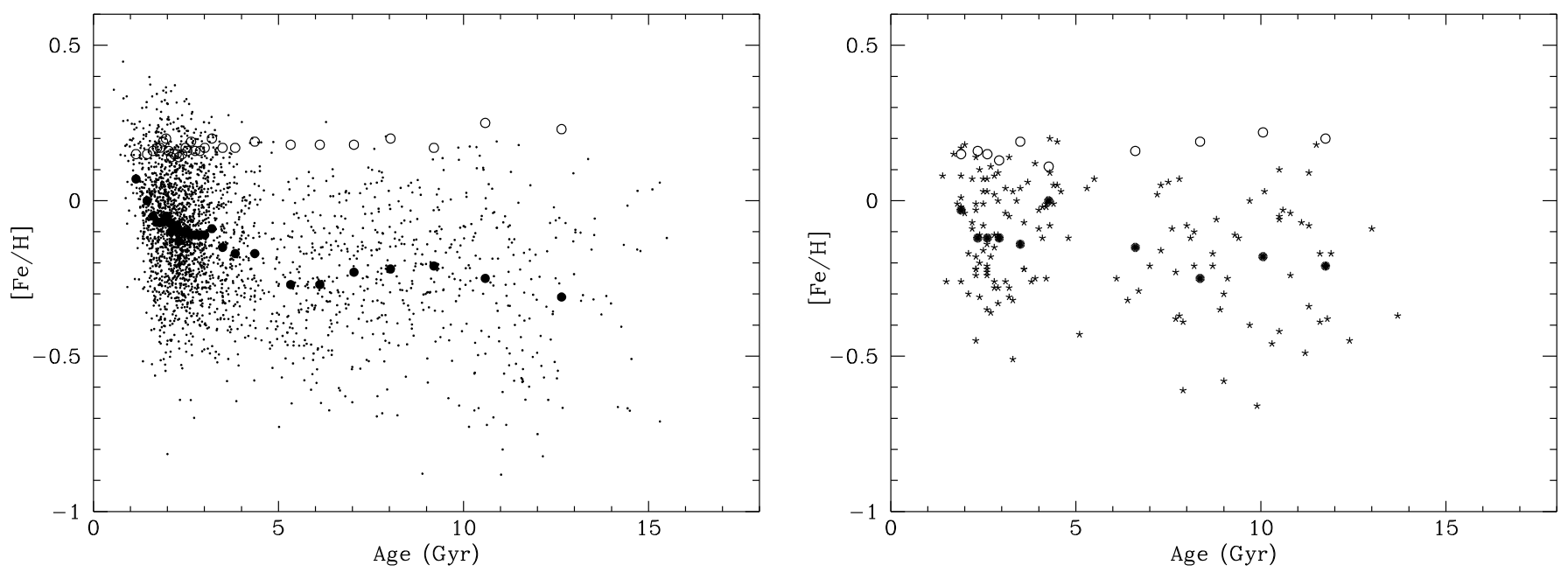

Fig. 4. Left: AMR for single stars with $\sigma_{\text {Age }}<25 \%$ (see text). Large filled dots show mean values, open circles the dispersions of [Fe/H] in bins with equal numbers of stars. Right: same, but for stars within $40 \mathrm{pc}$.
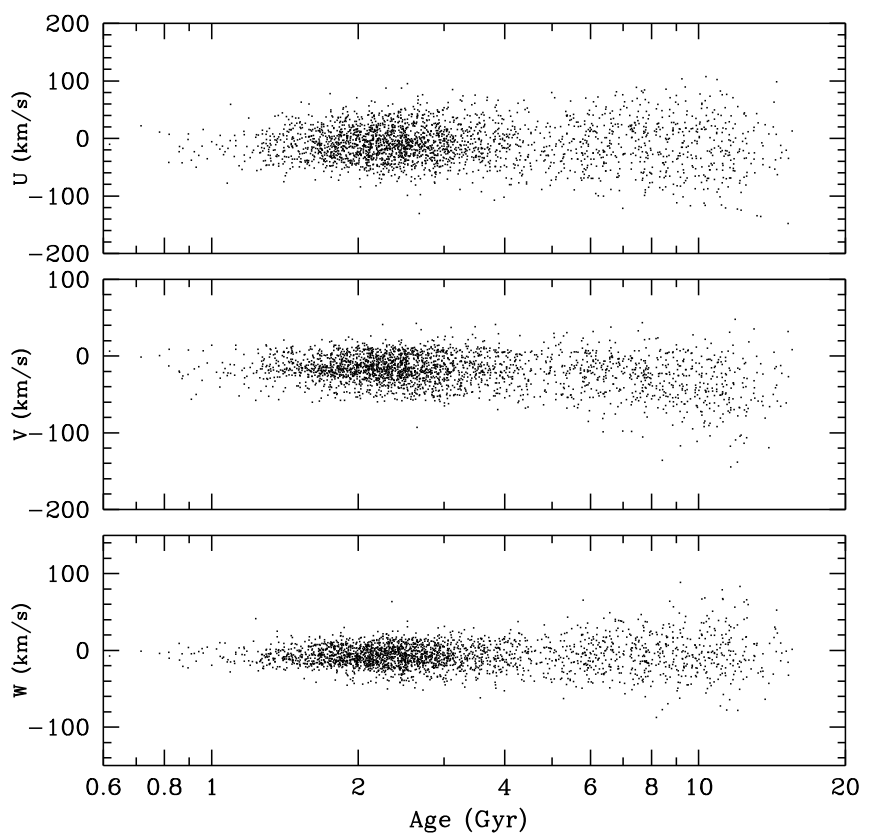

Fig. 5. $U, V$ and $W$ velocities vs. age for the 2626 single stars with $\sigma_{\text {Age }}<25 \%$.

evolution models with the real Milky May. We show it with our new data in Fig. 4 (left) for the subsample of stars defined above.

The apparent excess of metal-rich young stars is partly due to chemically peculiar Fm and Fp stars that cannot be identified from uvby $\beta$ photometry alone. As discussed in GCS I-II, it may reflect a preponderance of luminous young stars from a large volume and over-corrected for reddening. Figure 4 (right) shows the AMR for the volume-limited sample within $40 \mathrm{pc}$; it appears to be consistent with this explanation. In both cases, the dispersion in $[\mathrm{Fe} / \mathrm{H}]$ is $\sim 0.20$ dex, nearly independent of age.

\subsection{Age-velocity relation and disk heating}

Figure 5 shows the observed space velocity components as functions of age for the subsample defined above, while Fig. 6 shows
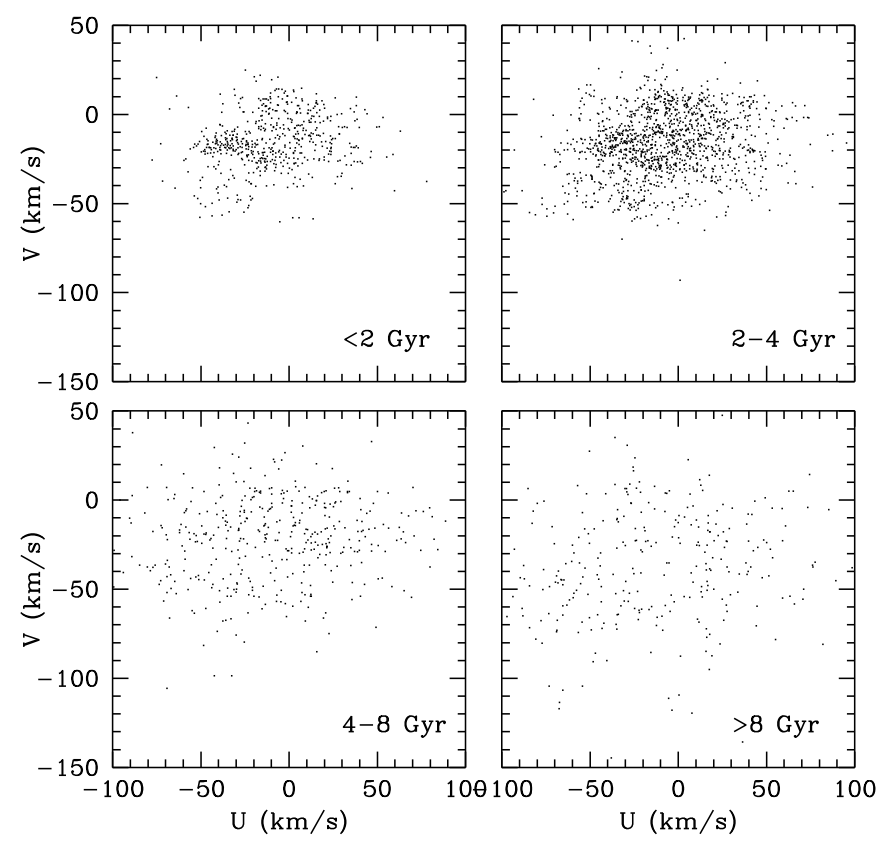

Fig. 6. $U-V$ diagram for the subsample of Fig. 5, separated into four age groups.

them in the $U-V$ plane, separated into four age groups. Like Figs. 20 and 30 of the GCS, they illustrate the significant substructure in the $U$ and $V$ velocity distributions that persists over a wide range of ages (see GCS I, Famaey et al. 2005; Seabroke \& Gilmore 2007; Bensby et al. 2007; and Antoja et al. 2008 for further discussion of these features). The revised velocity data do not significantly change the $W$ velocity distributions shown in GCS II (Fig. 32).

Figure 7 shows the resulting AVR for our observed sample of single stars, showing a smooth, general increase of the velocity dispersion with time in both $U, V$, and $W$. Fitting power laws while excluding the three youngest and three oldest bins, we find exponents of $0.39,0.40,0.53$ and 0.40 for the $U, V, W$ and total velocity dispersions - very similar to the values derived in GCS II. 

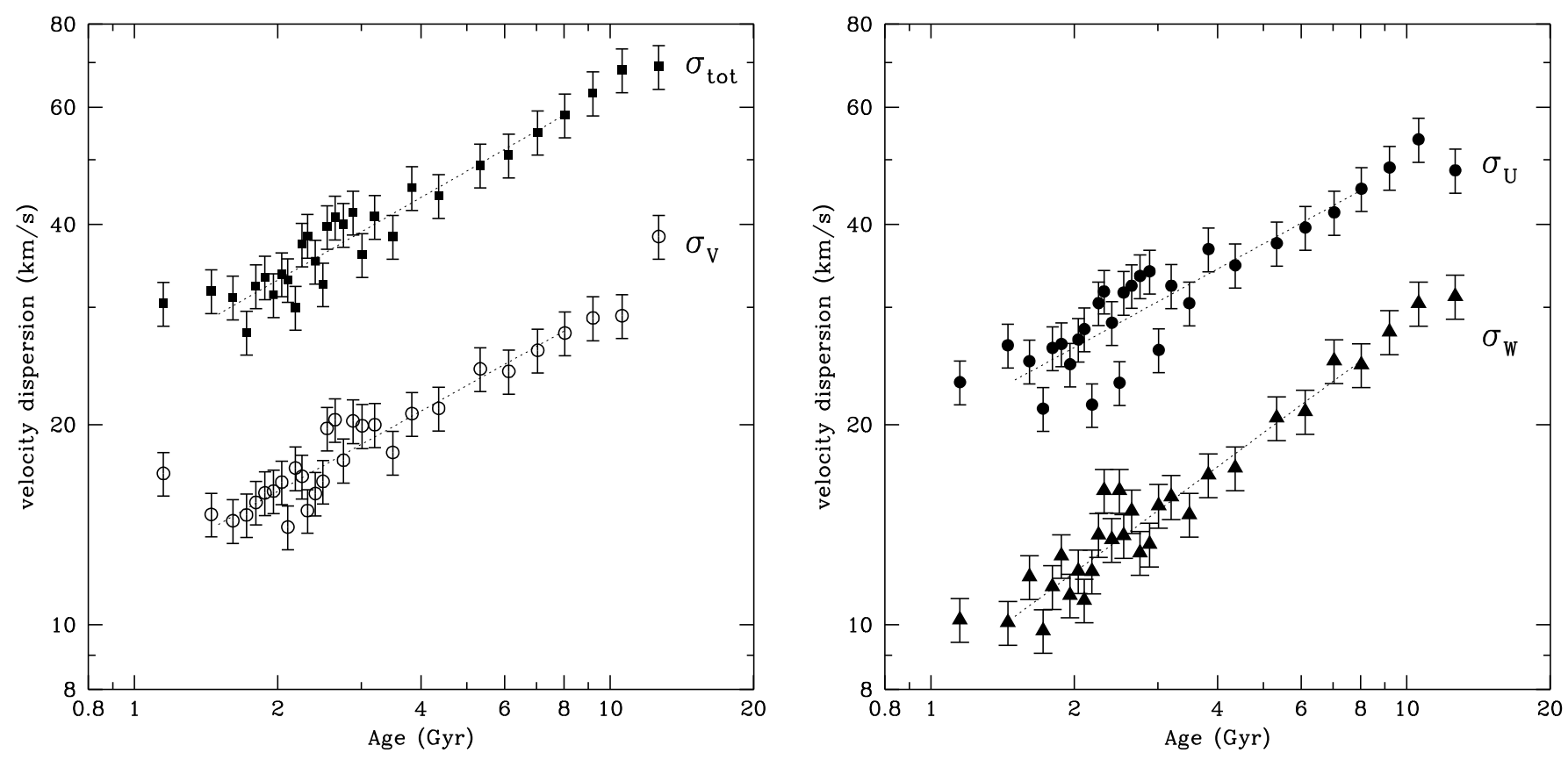

Fig. 7. Velocity dispersions vs. age for the subsample with $\sigma_{\text {Age }}<25 \%$. The 30 bins have equal numbers of stars ( 88 in each); the lines show fitted power laws. The 3 youngest and oldest bins were excluded from the fit.
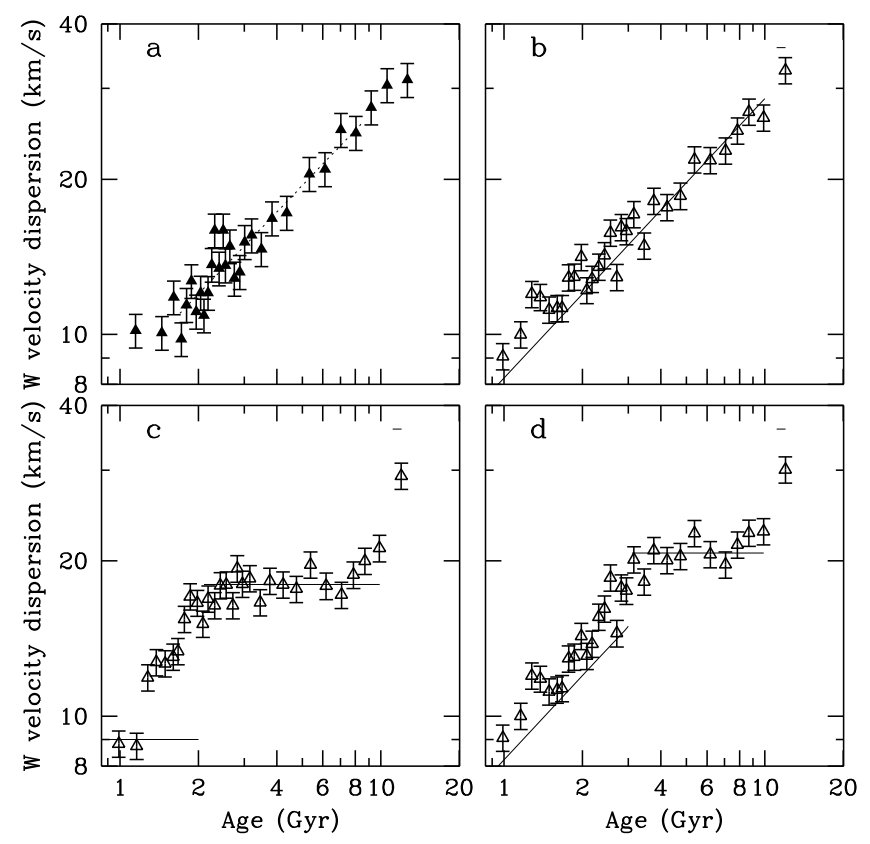

Fig. 8. a): observed AVR in $W$ (Fig. 7) with the fitted power law. b-d): simulated AVRs for three different disk heating scenarios (see text). Open symbols: rederived ages and velocity dispersions for the synthetic stars (sampling as in a); $\sigma_{\text {Age }}<25 \%$ ).

In GCS II we used simulations to check if our age determination process might change the shape or slope of the AVR (GCS II Fig. 34). We found this not to be the case when assuming a smooth increase in velocity over the whole lifetime of the disk, consistent with the observed AVR.

However, the coarse sampling of the AVR as shown in GCS I (Fig. 31) has led to suggestions that the data might equally well be described by an initial increase in velocity dispersion followed by a plateau. We have explored some of these possibilities through simulations following the recipe in GCS II. A "true" AVR is assumed, after which we compute synthetic "observations" with realistic random errors for a synthetic sample with similar astrophysical parameters as the real sample. The AVR is then reconstructed from the synthetic "observations" in the same manner as for the real data, focusing only on the $W$ component for the reasons discussed by Seabroke \& Gilmore (2007). The results of the simulations are compared to the observations in Fig. 8, panel $a$ repeating the observed $\sigma_{W}$ from Fig. 7.

The following three cases were considered:

The first synthetic AVR (panel $b$ ) is a continuous rise in velocity dispersion over the whole lifetime of the thin disk. However, simulations (Hänninen \& Flynn 2002) have shown that if only known local heating agents are assumed (i.e. GMCs), implausible amounts are needed to match the observed $\sigma_{W}$ for the oldest disk stars.

The second synthetic AVR (panel $c$ ) starts out cold until an age of $2.0 \mathrm{Gyr}$, then saturates at constant $\sigma_{W}=18 \mathrm{~km} \mathrm{~s}^{-1}$. This case is similar to the relation derived by Quillen \& Garnett (2001) from the sample of only 189 stars from Edvardsson et al. (1993).

The third assumed AVR (panel $d$ ) has a $\sigma_{W}$ increasing smoothly to $\sim 15 \mathrm{~km} \mathrm{~s}^{-1}$ at an age of $3 \mathrm{Gyr}$ when it rises abruptly to $\sim 21 \mathrm{~km} \mathrm{~s}^{-1}$, then remains constant until the maximum age of the thin disk at $10 \mathrm{Gyr}$. The scenario here is a late minor merger causing a step increase in $\sigma_{W}$. After the merger, the local heating processes cease to be effective for the stars formed prior to the merger, and $\sigma_{W}$ stays flat.

In all three simulations the thick disk appears at the age 11-12 Gyr, with a $\sigma_{W}$ of $36 \mathrm{~km} \mathrm{~s}^{-1}$ (short horizontal line above the last symbol in Panels $b-d$ ).

With the size and other properties of the sample we have analysed, there is a clear qualitative difference between the AVR corresponding to the three scenarios. However, a rigorous 
statistical analysis - which is beyond the scope of this paper would be required to establish solid confidence limits on any claim that these differences are indeed real. A much larger sample of stars with observational data of similar quality and completeness as those discussed here would make the picture much more clear-cut.

The continuing rise in $\sigma_{W}$ throughout the life of the disk with a higher slope than the in-plane velocity dispersions, (Fig. 7) remains a robust feature of the AVR. We note that a recent model (Schönrich \& Binney 2008) explains this as a natural consequence of the radial migration of stars in the disk, moving stars with hotter vertical kinematics from the inner disk into the solar neighbourhood. This stellar migration at the same time causes the large scatter in $[\mathrm{Fe} / \mathrm{H}]$ for stars of a given age. Schönrich \& Binney (2008) utilise a coupled chemical-kinematical evolution model to explain the observed relations, seen already in GCS I. Another model along the same lines, but using a different method ( $N$-body+SPH) is described by Roškar et al. (2008).

\section{Conclusions}

Implementing the new and more accurate Hipparcos parallaxes is a clear improvement of the observational data for the GCS sample. Our thorough review of the steps leading to the astrophysical parameters suggests that further major improvement is unlikely unless substantially more accurate high-resolution spectroscopy, multicolour photometry and/or parallaxes become available for the complete sample, e.g. from Gaia.

The single main avenue for progress in the interim is an improved calibration of effective temperature from the existing data. With long-baseline optical interferometers now in routine use, much progress is possible from new, accurate measurements of angular diameters of FG dwarfs, combined with correspondingly accurate bolometric fluxes. In the best of worlds, new 3D, hydrodynamic, NLTE model atmospheres will then also resolve the current difference between spectroscopic and photometric determinations of $T_{\text {eff }}$ and its repercussions on the derived [Fe/H] values. In the meantime, we prefer the scale based on the fundamental empirical data.

Apart from improvements in the input data, the determination of isochrone ages now appears to be a robust technique giving substantially consistent results if appropriate precautions are taken, as discussed in Sect. 6.1. Indeed, the basic features of the age-metallicity and age-velocity relations (slopes, dispersion) have remained essentially unchanged through the various revised calibrations discussed in GCS I-III. We note with interest that the current trend in models for the Galactic disk is to identify the mechanisms that may be responsible for these robust features.

Acknowledgements. We reiterate our thanks to our many collaborators on the original GCS from Observatoire de Genève, Harvard-Smithsonian Center for Astrophysics, ESO, and Observatoire de Marseille. It was made possible by large grants of observing time and travel support from ESO, the Danish Board for Astronomical Research, and the Fonds National Suisse pour la Recherche Scientifique. We also gratefully acknowledge the substantial financial support from the Carlsberg Foundation, the Danish Natural Science Research Council, the Smithsonian Institution, the Swedish Research Council, the Nordic Academy for Advanced Study, and the Nordic Optical Telescope Scientific Association. We have made use of the SIMBAD and VizieR databases, operated at CDS, Strasbourg, France.

\section{References}

Antoja, T., Figueras, F., Fernandez, D., \& Torra, J. 2008, A\&A, 490, 135 Baines, E. K., McAlister, H. A., ten Brummelaar, T. A., et al. 2008, ApJ, 680, 728

Boyajian, T. S., McAlister, H. A., Baines, E. K., et al. 2008, ApJ, 683, 424 di Benedetto, G. P. 1998, A\&A, 339, 858

Bensby, T., Oey, M. S., Feltzing, S., \& Gustafsson, B. 2007, ApJ, 655, L89

Blackwell, D. E., \& Lynas-Gray, A. E. 1994, A\&A, 282, 899

Casagrande, L., Portinari, L., \& Flynn, C. 2006, MNRAS, 373, 13

Crawford, D. L. 1975, AJ, 80, 955

Crawford, D. L., \& Barnes, J. V. 1969, AJ, 74, 407

Crawford, D. L., \& Perry, C. L. 1966, AJ, 71, 206

Demarque, P., Woo, J., Kim, Y., \& Yi, S. K. 2004, ApJS, 155, 667

Edvardsson, B., Andersen, J., Gustafsson, B., et al. 1993, A\&A, 275, 101

Famaey, B., Jorissen, A., Luri, X., et al. 2005, A\&A, 430, 165

Flynn, C., Sommer-Larsen, J., \& Christensen, P. R. 1996, MNRAS, 281, 1027

Girardi, L., Bressan, A., Bertelli, G., \& Chiosi, C. 2000, A\&AS, 141, 371

Holmberg, J., Nordström, B., \& Andersen, J. 2007, A\&A, 475, 519 (GCS II)

Hänninen, J., \& Flynn, C. 2002, MNRAS, 337, 731

Jenkins, J. S., Jones, H. R. A., Pavlenko, Y., et al. 2008, A\&A, 485, 571

Jørgensen, B. R., \& Lindegren, L. 2005, A\&A, 436, 127

Nordström, B., Mayor, M., Andersen, J., et al. 2004, A\&A, 418, 989 (GCS I)

North, J. R., Davis, J., Bedding, T. R., et al. 2007, MNRAS, 380, 80

North, J. R., Davis, J., Robertson, J. G., et al. 2009, MNRAS, accepted [arXiv:0811.1813]

Olsen, E. H. 1984, A\&AS, 57, 443

Quillen, A. C., \& Garnett, D. 2001, in Galaxy Disks and Disk Galaxies, ed. J. G.

Funes, S. J., \& E. M. Corsini, ASP Conf. Ser., 230, 87

Ramírez, I., \& Meléndez, J. 2005, ApJ, 626, 446

Roškar, R., Debattista, V. P., Quinn, T. R., Stinson, G. S., \& Wadsley, J. 2008, ApJ, 684, L79

Salasnich, B., Girardi, L., Weiss, A., \& Chiosi, C. 2000, A\&A, 361, 1023

Schönrich, R., \& Binney, J. 2008, MNRAS, submitted [arXiv:0809.3006v1]

Seabroke, G. M., \& Gilmore, G. 2007, MNRAS, 380, 1348

Sousa, S. G., Santos, N. C., Mayor, M., et al. 2008, A\&A, 487, 373

Takeda, G., Ford, E. B., Sills, A., et al. 2007, ApJS, 168, 297

VandenBerg, D. A., Bergbusch, P. A., \& Dowler, P. D. 2006, ApJS, 162, 375

van Leeuwen, F. 2007, A\&A, 474, 653 